Didáctica Geográfica n 21, 2020,pp. 227-246

DOI: https://doi.org/10.21138/DG.551

ISSN electrónico: 2174-6451

\title{
TRABAJO DE CAMPO URBANO: UNA ESTRATEGIA DI- DÁCTICA DE LA GEOGRAFÍA PARA LA ENSEÑANZA PRO- YECTUAL DEL PAISAJE EN EL ÁMBITO UNIVERSITARIO
}

URbAN FIELD WORK: A DIDACTIC STRATEgy OF GEOGRAPHY FOR PROJECTIVE LANDSCAPE TEACHING IN THE UNIVERSITY ENVIRONMENT

Travail urbain Sur le terrain: Une stratégie didactique de la GÉOgRAPHIE POUR L'ENSEIGNEMENT PAYSAger PROJECTIF EN MILIEU UNIVERSITAIRE

Gabriela Eda Campari

Universidad de Buenos Aires, Argentina

gcampari@fadu.uba.ar

Recibido: 04/06/2020

Aceptado 29/09/2020

\section{RESUMEN:}

El objetivo del artículo es analizar el Trabajo de Campo Urbano (TCU) como estrategia didáctica de la asignatura Geografía de la Licenciatura en Planificación y Diseño del Paisaje, carrera de grado de la Universidad de Buenos Aires. En lo metodológico, se examinan el TCU dentro de la trayectoria del Trabajo Final Integrador del curso y testimonios de estudiantes colectados mediante encuestas. Como conclusión, la experiencia permite al alumnado potenciar el conocimiento del paisaje urbano y comprender la relevancia de articular las prácticas y percepciones comunitarias a su abordaje proyectual. 


\title{
Palabras clave:
}

Didáctica de la Geografía; diseño del paisaje; observación participante.

\begin{abstract}
:
The objective of the article is to analyze the Urban Field Work (UFW) as a didactic strategy of the Geography subject of the Degree in Landscape Planning and Design, undergraduate degree at the University of Buenos Aires. From a methodological point of view, the UFW is examined within the course of the Integrative Final Work of the course and testimonies from students collected through surveys. In conclusion, the experience allows students to enhance their knowledge of the urban landscape and understand the relevance of articulating community practices and perceptions to their project approach.
\end{abstract}

\section{KEWORDS:}

Geography didactics; landscape design; participant observation.

\section{RÉSUMÉ:}

L'objectif de ce travail est d'analyser le travail de terrain urbain (TTU) en tant que stratégie didactique du sujet de géographie du diplôme en planification et conception du paysage, diplôme de premier cycle à l'Université de Buenos Aires. D'un point de vue méthodologique, le TTU est examiné dans le cadre des Travaux Finaux Intégratifs du cours et des témoignages d'étudiants recueillis au travers d'enquêtes. En conclusion, l'expérience permet aux étudiants d'approfondir leurs connaissances du paysage urbain et de comprendre la pertinence d'articuler les pratiques et les perceptions communautaires à leur approche de projet.

\section{Mots-CLÉS}

Didactique géographie; Aménagement paysager; observation des participants. 


\section{INTRODUCCIÓN}

El Trabajo de Campo, desde la mirada geográfica, constituye una propuesta didáctica que permite integrar teoría y empiria, la lectura y comprensión crítica del paisaje urbano y su enseñanza proyectual; un "dispositivo pedagógico-instrumental" (Souto, 2006) que comprende una serie de tareas y acuerdos respecto a tiempos, recursos y objetivos; un espacio promotor de cambios e innovaciones. Piñeiro Peleteiro define Trabajo de Campo como "...cualquier actividad realizada por el profesor y los alumnos sobre el terreno" (2013 [1997], p. 25) y Zusman (2011) sostiene que, actualmente, el trabajo de campo clásico y el etnográfico integran una única labor, en la cual la observación visual emerge como una etapa de conocimiento del terreno y la interacción con la comunidad permite explorar e incorporar su perspectiva y aspectos vinculados con el habitar. Las actividades de observación directa del paisaje, según Sánchez Ogallar, acontecen como una secuencia de "síntesis-análisis-síntesis" que parte de una visión general con clara impronta subjetiva, continúa con el análisis y estudio de sus elementos y relaciones y retorna a una “...visión sintética, (...) más coherente y organizada” (2013 [1997], p. 52). La dimensión participante del Trabajo de Campo, permite explorar alternativas proyectuales viables en relación a las demandas específicas de una comunidad y el campo se torna "una instancia necesaria de aproximación a los sujetos, que entraña una reciprocidad de comunicación y de sentidos...” (Guber, 2004, p. 184), que “...no solo implica la posibilidad de observar, interactuar e interpretar a los actores en [su] contexto (...) y hacerlo durante un tiempo prolongado, sino también de participar en las múltiples actividades que (...) despliegan en su vida cotidiana. Una instancia fundamental para la comprensión de las relaciones sociales (...) un ámbito en el que interactúan sujetos, se comparten significados y se explicitan múltiples prácticas sociales y simbólicas" (Vasilachis de Gialdino, 2006, p. 117).

Entendemos el paisaje como "cualquier parte del territorio tal como la percibe la población, cuyo carácter sea el resultado de la acción y la interacción de factores naturales y/o humanos" (Consejo de Europa, 2000), en cuya fisonomía se imprimen diversos tiempos históricos a modo de "palimpsesto" (Santos, 1996) que exhiben las formas de producción de objetos y espacios. El paisaje, se define por sus formas, por su morfología (Dollfus, 1978) y, según Nogué (2009), constituye un "constructo social" complejo donde intervienen las múltiples miradas y maneras de apropiación y valoración que una sociedad proyecta en la naturaleza, “....una determinada forma de organizar y experimentar el orden visual de los objetos geográficos en el territorio” (p. 12). Estos modos de observar, percibir y descubrir el paisaje ponen en relieve aspectos vinculados al estudio de las prácticas, representaciones, interacciones y problemáticas sociales que se producen y reproducen en él. El paisaje urbano, según Estébanez (1995), responde a su entorno natural -situación y emplazamiento-, a sus componentes físicos, usos del 
suelo y trama edificada y al modo en que la población percibe esos componentes y el rol que desempeñan en razón de su valoración para el sentido de pertenencia, la identidad y el ambiente.

Enseñar paisaje, según Liceras Ruiz (2018) implica una formación de la mirada, cognitiva, estética, ética y social, aspectos que complejizan su realidad y dan cuenta de su importancia como recurso educativo. Proyectar el paisaje, siguiendo a Besse, refiere “...ponerlo en imágenes o representarlo (proyección) e imaginar lo que podría ser o llegar a ser (proyectación)" (2006, p.168), instancias de la enseñanza-aprendizaje proyectual que pueden verse acotadas si únicamente se estimula diseñar "lo nuevo" y no la aprehensión vivencial del paisaje. La propuesta pedagógica de la asignatura Geografía $^{1}$ de la Licenciatura en Planificación y Diseño del Paisaje de la Universidad de Buenos Aires articula las clases teórico-prácticas del aula-taller con el Trabajo de Campo Urbano, especialmente en áreas verdes públicas, a fin de que el alumno las recorra, interactúe con los actores sociales y obtenga, mediante técnicas cuantitativas y cualitativas, información sobre las prácticas y percepciones comunitarias, a fin de integrarlas al abordaje proyectual y la producción de propuestas paisajísticas para espacios abiertos urbanos, ejercitando así un proceso cognitivo desde los intercambios, diferencias y desafíos experimentados con el "otro".

En este sentido, la asignatura implementa un abordaje geográfico del paisaje urbano, de las mutaciones territoriales, desigualdades en su apropiación y discriminación en su uso desde una dimensión social y crítica (Ortega Valcárcel, 2007) y recurre a la didáctica, en tanto campo práctico de la enseñanza, como “...una gama variable de conocimientos que abarca principios teóricos, modelos comprensivos, reglas prácticas, métodos y estrategias..." (Feldman, 1999, p. 25). Sus configuraciones comprenden el modo particular del docente para desarrollar su clase, entramando situaciones, recortando contenidos, proponiendo líneas de trabajo y procesos metacognitivos. Un buen cauce de estas configuraciones es aquel que promueve procesos reflexivos y rupturas con el conocimiento cotidiano y, a la vez, transmite nuevos saberes disciplinares.

En este contexto, el objetivo del artículo es analizar el Trabajo de Campo Urbano (TCU) como estrategia didáctica, en el ámbito universitario, para potenciar el conocimiento del paisaje urbano y comprender la relevancia de articular las prácticas y percepciones comunitarias a su abordaje proyectual.

${ }^{1}$ La asignatura es cuatrimestral, obligatoria, con examen final e integra el $3^{\circ}$ nivel del Plan de Estudios de la carrera que tiene título interdisciplinar y es dictada entre las Facultades de Agronomía y de Arquitectura, Diseño y Urbanismo. 


\section{METODOLOGÍA}

Desde el punto de vista metodológico, indagaremos la trayectoria de la experiencia del Trabajo de Campo Urbano (TCU) dentro del proceso de enseñanza-aprendizaje de la asignatura, mediante una revisión bibliográfica desde las ciencias sociales, la compilación e indagación de los antecedentes, propuestas y recursos didácticos vinculados a su desarrollo y del TIF como instancia de aprobación de la asignatura y el análisis de testimonios de los estudiantes acerca de aspectos de la experiencia de campo. El TIF es la producción escrita y gráfica del estudio de caso que los estudiantes presentan y defienden en la instancia de evaluación final, donde dan cuenta de los conocimientos y habilidades adquiridos para recopilar y analizar información y antecedentes, identificar aspectos geográficos y problemáticas, diseñar instrumentos de registro y relevamiento de datos en campo y generar un diagnóstico para formular una propuesta de diseño paisajístico del área de estudio.

Para determinar la ubicación geográfica y tipología del espacio verde elegido por los estudiantes como ámbito para el desarrollo de su experiencia de campo urbano y propuesta proyectual, recurriremos al análisis de 77 TIF-caso presentados en el período 2006-2016, universo que, asimismo, utilizaremos para definir los tiempos de presentación de los TIF en relación con la oportunidad de realización del TCU.

Asimismo, se explorarán los testimonios vinculados al TCU de 82 estudiantes universitarios, obtenidos mediante las respuestas brindadas a una encuesta de opinión, de manera anónima, al finalizar el cursado de la asignatura durante los períodos 20122013 y 2015-2017. El instrumento para colectar los testimonios se conformó por tres preguntas abiertas en las que los estudiantes se refirieron a: 1) Distintos aspectos de la asignatura -entre ellos el Trabajo de Campo- y que se considera en este artículo; 2) Los contenidos posibles a incorporar al programa de estudio y 3) Las temáticas propuestas para ser desarrolladas con mayor amplitud. Por último, la encuesta contaba con un apartado para el aporte de otras sugerencias por parte de los estudiantes.

\section{EXPERIENCIAS URBANAS DE CAMPO}

El Trabajo de Campo Urbano de la asignatura, previo al año 2004, se desarrollaba finalizado el curso y fuera del período lectivo como parte del TIF. Los estudiantes conformaban grupos y elegían, previo acuerdo en clase con el cuerpo docente, un espacio verde urbano como estudio de caso, aplicando en la labor de campo los contenidos teóricos trabajados y una serie de consignas vinculadas con su abordaje, dado que el equipo docente no participaba de la experiencia. El espacio debía contar con características de cercanía y cotidianeidad y permitir realizar las actividades del trabajo de campo -observar, recorrer, registrar e interactuar con sus usuarios-, para obtener los insumos para la posterior problematización, análisis, diagnóstico y elaboración de una propuesta paisajística viable. 
Con la finalidad de que los alumnos contarán con una experiencia del TCU previa a la que debían llevar a cabo en el TIF, durante 2004-2005 se programó, en el $2^{\circ}$ bimestre del curso, una práctica piloto denominada "Circuito Parque de los Patricios", ${ }^{2}$ un itinerario impreso articulado en función de hitos barriales del patrimonio histórico de la Ciudad de Buenos Aires, cuyo recorrido efectuaban a pie el docente a cargo y los alumnos; decisión que resultó un punto de inflexión y facilitó el abordaje del TCU en la instancia extracursada del TIF y dio lugar a las experiencias que seguidamente se describen.

\subsection{Fase decisoria I: El recorrido empieza por acá...}

A partir de 2006 y hasta 2010 (Figura 1), el Trabajo de Campo Urbano continuó realizándose en el $2^{\circ}$ bimestre y, en el marco de una relectura del paisaje de los espacios verdes del sur de la Ciudad de Buenos Aires, incorporó "Circuitos de Interpretación" en los barrios de Parque Patricios y Barracas, ${ }^{3}$ con el propósito de considerar esas áreas no sólo “...desde una perspectiva asociada con (...) su recuperación material, sino inclu[yendo] una mirada que permitiera entenderlos como productos sociales..." (Varela y Campari, 2012, p. 321). Los circuitos, diseñados por el equipo docente en relación al paisaje de la "Ciudad y sus espacios verdes como texto", brindaron una cartilla más amplia y dotada de planos e imágenes que permitió a los estudiantes recorrerlos e incorporar al análisis de las áreas verdes aspectos del paisajismo urbano y de los procesos históricos vinculados a su génesis y configuración territorial.

En tal sentido, se definieron los siguientes circuitos:

1. Circuito A: "Lo peligroso" de los bordes. Lejos del centro de la Ciudad entre la quema, la peste, los presos y el lugar de los hospitales públicos.

2. Circuito B: La interconexión en ambas márgenes del Riachuelo a través de los circuitos económicos: caminos y puentes.

3. Circuito C: La construcción destinada a los barrios obreros y el lugar del "espacio verde": vivienda social y educación.

4. Circuito D: La degradación ambiental y la reconversión de los espacios verdes.

Cada circuito contaba en su itinerario con la marcación de objetos-forma del paisaje urbano de distintas etapas cronológicas. En su recorrido, a pie, la docente a cargo guiaba, transmitía las consignas e indicaba los hitos, a fin de que los alumnos observaran e identificaran la coexistencia en el paisaje de diferentes tiempos yuxtapuestos e interactuantes

2 El "Parque de los Patricios" es un espacio verde público concebido, a principios del siglo XX, bajo la impronta de saneamiento y ornato, como sitio de esparcimiento del barrio Parque Patricios.

3 Dichos barrios integran, junto a los de La Boca y Nueva Pompeya, la Comuna 4. 
(Santos, 1996) y registraran sus experiencias y percepciones, las que luego retomaban en el aula-taller. En 2010 se efectuó una revisión y ajuste del TCU, impartiéndose en las primeras clases las consignas para desarrollar el TIF. Dado que el diagnóstico y proyecto paisajístico continuaban desarrollándose finalizado el curso y el ciclo lectivo anual, se decidió brindar a los alumnos tutoriales previos a su producción y que éstos presentaran un diagnóstico preliminar, antes de culminar la cursada, a fin de llevar a cabo una devolución. Por último, se reformuló la propuesta de los circuitos, en cuanto a su número, temática y tiempos de recorrido y para realizar las tareas de observación y registro.

\subsection{Fase transicional II: Cambios de escala, vínculos con la comunidad}

En 2011 el Trabajo de Campo Urbano continuó en el $2^{\circ}$ bimestre del curso y, a fin de analizar los espacios verdes sitos en instituciones públicas locales de atención de la salud, se recurrió para su implementación al Circuito de Interpretación "A", incluyendo el acceso al predio del Hospital de Infecciosas "Doctor Francisco Javier Muñiz"4, establecimiento pabellonal con destacada presencia de áreas verdes internas (Figura 1). Al año siguiente, la práctica adicionó el relevamiento cuali-cuantitativo del arbolado del nosocomio, dado los deterioros sufridos por un temporal en abril de 2012. En esta oportunidad, el caso de estudio fue para todos el espacio verde del Muñiz, solapándose las actividades de la experiencia didáctica de campo durante el curso con las realizadas para elaborar el TIF fuera de la cursada, tal como ocurrió con las entrevistas y encuestas a sus usuarios donde los alumnos pusieron “...a prueba sus habilidades para abordar un caso complejo y su capacidad de interactuar con la comunidad hospitalaria e interpretar las problemáticas diarias" (Campari, 2014, p. 89). A mitad del curso se incorporó un intercambio con alumnos de la asignatura del ciclo lectivo anterior en el que expusieron sus TIF aprobados y evacuaron inquietudes. La experiencia didáctica brindó así andamiaje para visibilizar y pensar el paisaje hospitalario (Campari, Giacoia, y Salerno, 2014) desde el ejercicio proyectual y su significado como área verde urbana en el contexto de planificación territorial.

\subsection{Fase aumentada III: Las mutaciones económicas-productivas del paisaje de Parque Patricios}

A partir de 2013 (Figura 1), en virtud de una serie de profundas mutaciones del paisaje de la Comuna 4, especialmente del barrio Parque Patricios, producto de una política de desarrollo distrital económico-productivo iniciada en 2008, se reformuló la propuesta didáctica con un circuito vinculado a las instituciones de salud pública (ver fase I y II,

${ }^{4}$ Ubicado en el barrio Parque Patricios. 
Circuito I) y otro en base al Circuito D de la fase I que, actualizado en sus contenidos, se denominó "Circuito II: De los Parques al Distrito Tecnológico".

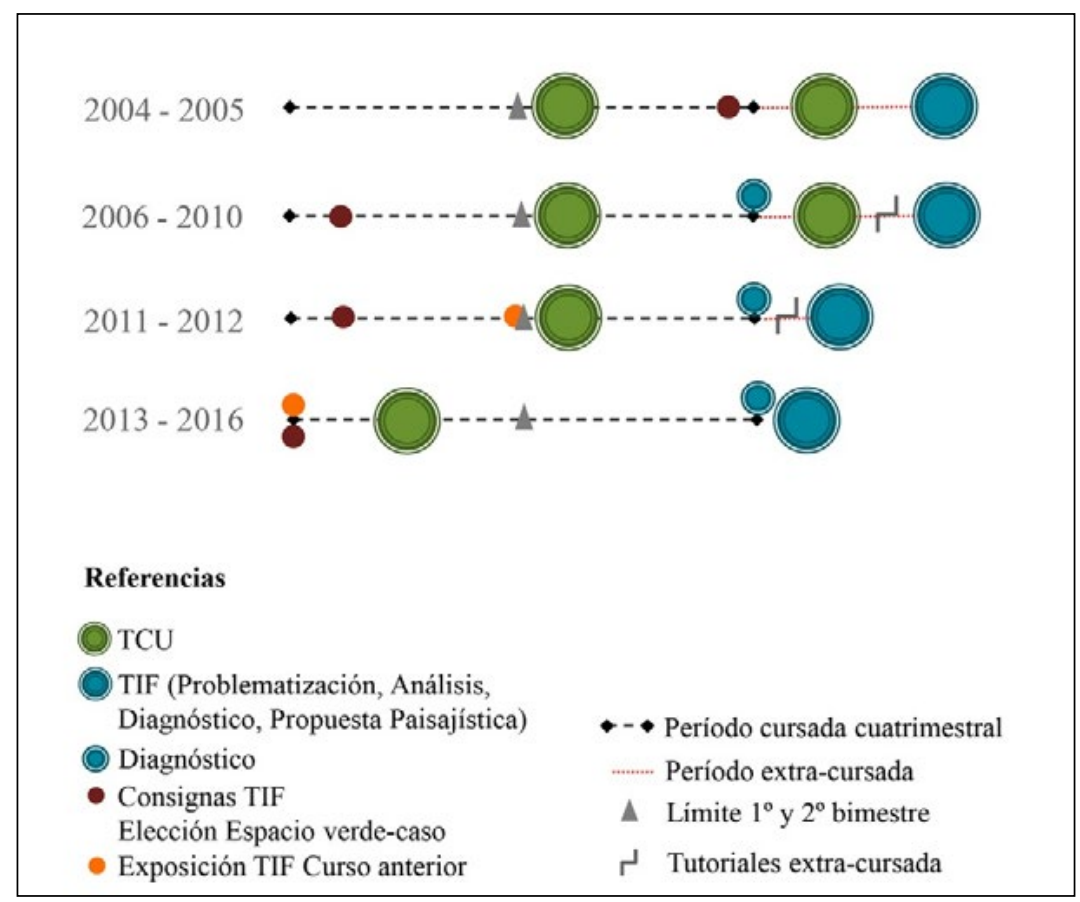

FIGURA 1. Esquema de experiencias de campo.

Fuente: Elaboración propia en base al archivo de la asignatura

A diferencia de años anteriores, la práctica didáctica del TCU se inició en el $1^{\circ}$ bimestre del curso y las consignas para el Trabajo Integrador Final se brindaron en la primera clase. Los alumnos volvieron a elegir el espacio verde urbano-caso para su TIF y se mantuvo la exposición de trabajos del año anterior, la presentación del diagnóstico en las últimas clases y la entrega de la propuesta paisajística en el examen final, pautas que continuaron hasta 2016 con la salvedad que, a partir de 2014, el intercambio y exposición de trabajos del ciclo anterior comenzó a realizarse en la primera clase para mejorar la comprensión y articulación de las tareas del TCU y del TIF.

\subsection{Recalculando...}

Documentar la trayectoria en la asignatura de esta experiencia de enseñanza permitió “...abrir el juego a la formulación de preguntas y da[r] lugar a la necesidad auténtica de 
comprender mejor...” (Libedinsky, 2001, p. 95) la labor docente y, a la vez, formular las siguientes consideraciones en relación a las fases I y $\mathrm{III}^{5}$ :

Los estudiantes presentaron dificultades para identificar y analizar problemáticas vinculadas al paisaje a fin de seleccionar su estudio de caso, circunstancia que asociamos a una falta de ejercicio en dichas tareas, derivada de una práctica educativa proveedora de problemáticas y áreas para llevar a cabo la propuesta proyectual, que no estimula la curiosidad y motivación para indagar e innovar; situación que se reflejó en algunos testimonios que planteaban: "Más salidas para entrenar la observación y ver en la práctica que cuestiones o relaciones son importantes para recabar del espacio público $y$ poder analizar" ". Los tipos de espacio verde elegidos mayoritariamente como área estudio, fueron las plazas $(34,43 \%)$ y los parques $(14,75 \%)$ que concentraron casi la mitad del número total de casos $(49,18 \%)$, correspondiendo el resto a otras tipologías $(50,82 \%)$, entre ellas, los boulevares y espacios verdes lindantes o insertos en áreas costeras, ferroviarias, educativas y conjuntos habitacionales. En cuanto a la ubicación geográfica de los casos, el 67,21\% del total correspondió a la Ciudad de Buenos Aires, el 26,23\% a la provincia de Buenos Aires y el 6,56\% a otras provincias de la República Argentina. ${ }^{7}$ En este último caso, cabe destacar que los espacios verdes se emplazaban en el lugar de residencia de los estudiantes, quienes desarrollaban las tareas de campo al retornar a sus domicilios con motivo del fin del ciclo lectivo.

Un inconveniente de elaborar el TIF fuera de la cursada fue la demora en su presentación, circunstancia que dilataba en el tiempo el momento de rendir el examen y aprobar la asignatura. En este sentido, los tutoriales programados por el equipo docente se veían interrumpidos, generando instancias fragmentadas que impactaban en los avances y producción del trabajo y la labor de seguimiento del docente-tutor. La presentación tardía del TIF se registró principalmente durante la fase I, en la que el 43,75\% de los trabajos se concretó a los dos años de que los estudiantes finalizaran la cursada situación que se revirtió de manera notoria durante la fase III, en la cual el 51,72\% de los TIF se presentaron dentro de los tres meses posteriores a completar el curso. Asimismo, cabe destacar que en la fase I tan sólo el 3,13\% de los estudiantes aprobó la materia en este último período citado

Los circuitos si bien incorporaron la práctica didáctica de campo durante el desarrollo del curso y contribuyeron al conocimiento - "Yo lo sentí como si fuera un tour, porque

\footnotetext{
${ }^{5}$ Las consideraciones se efectúan sobre 61 TIF-caso de las fases I y III. La etapa transicional de la Fase II se integró por 16 TIF que abordaron el espacio verde del Hospital Muñiz como estudio de caso.

6 Encuesta 03 (2015).

${ }^{7}$ Los casos se ubicaron en las provincias de Jujuy, Córdoba, Corrientes, Misiones y Río Negro.
} 
recorrías las zonas conociendo de ellas" ${ }^{-}$, al estandarizarse ciertas consignas-guía, devinieron en una propuesta que terminó por no estimular la creatividad ni la aptitud de los grupos de trabajo para asumir desafíos proyectuales desde una perspectiva crítica y social del paisaje. Asimismo, el hecho que los estudiantes dilataran la realización del TCU correspondiente al TIF, provocó su desarticulación con la labor llevada a cabo en la cursada, situación que tuvo eco en algunos testimonios y puso en evidencia la necesidad de anticipar su inicio - "Creo que el trabajo debería empezar antes porque es poco el tiempo para elaborarlo" - y de vincular el desarrollo de ambas experiencias -¿Qué propondría para el Trabajo de Campo? "poder relacionarlo con el estudio de caso" ${ }^{10}$-.

En cuanto a la elección del espacio verde-caso para llevar a cabo el TCU en el contexto del TIF, observamos que los estudiantes la hacían en función de su propia imagen ambiental, de parcialidades y fragmentos relacionados con lo vivido (Lynch, 1998), en la que se reflejaban los paisajes cotidianos de su experiencia personal, de su interacción con el entorno inmediato y su realidad social, donde “...la valoración de un paisaje y (...) en su capacidad de atracción, pesan decisivamente consideraciones emocionales y estéticas que dependen de la percepción selectiva..." (Capel y Urteaga, 1982, p. 44). Este recorte construía su propio recorrido y la selección de casos recaía en áreas verdes ya exploradas o trabajadas en otras asignaturas proyectuales, situación que exhibía: a) una noción acotada del paisaje, vinculada principalmente a prácticas recreativas y circulatorias en el espacio público, b) cierta dificultad para entender y relacionar el paisaje con espacios degradados, marginales o invisibilizados, es decir, que no respondieran estrictamente a determinados parámetros de ornato vigentes (Varela y Campari, 2009), y c) una limitación para estimular el desafío de abandonar la zona de confort y abordar contextos sociourbanos desconocidos, tales como el espacio verde hospitalario, visualizándose “...mиy interesante aprender a diseñar un espacio, escuchando y entendiendo las necesidades del usuario; (...) [lo que] hace más sencillo diseñar" ${ }^{11}$.

Repensar la articulación del curso con el Trabajo Integrador Final y el Trabajo de Campo Urbano como nexo, condujo a interrogarnos respecto al modo de activar líneas de acción que estimularan la resolución de actividades y profundizaran la búsqueda y extrapolación de conocimientos a situaciones complejas de análisis del paisaje. La asignatura requería promover durante el aprendizaje conductas proactivas de los alumnos en su tarea grupal y con el equipo docente y ampliar la indagación y comprensión del medio físico y social para alcanzar diagnósticos que desencadenaran propuestas viables,

\footnotetext{
8 Encuesta 02 (2015).

9 Encuesta 01 (2013).

${ }^{10}$ Encuesta 01 (2015).

${ }^{11}$ Encuesta 08 (2012).
} 
por lo que a tales fines establecimos ajustes y nuevos criterios para realizar el TCU en relación intrínseca con el Trabajo Integrador Final, los que describimos seguidamente.

\subsection{Innovar en la propuesta didáctica}

A partir de 2017 la experiencia didáctica del TCU, si bien mantuvo su zona de anclaje geográfico en el sur de la Ciudad de Buenos Aires, no ofreció a los alumnos un circuito barrial preestablecido. En cuanto al caso de estudio, el equipo docente identificó los espacios verdes públicos emplazados en la Comuna 4 y verificó, entre otros aspectos, sus condiciones de accesibilidad, afectaciones materiales limitantes y potencialidad de análisis, efectuando un recorte que dio cuenta de sus tipologías y generó un listado de 11 áreas que puso a disposición de los grupos en la primera clase. Cada grupo, tras un debate interno, escogió tres espacios del listado en un orden de preferencia y, posteriormente, lo dio a conocer. El equipo docente asignó los casos de estudio considerando el área elegida en primer término por cada grupo, resolviendo las coincidencias en esa posición mediante la deliberación y consenso intergrupal, mecanismo que enriqueció los fundamentos y dinámica del proceso de selección.

A partir de fichas didácticas de clase y bibliografía temática, cada grupo definió los aspectos del caso de estudio objeto de observación y registro, los que incorporaron a una síntesis gráfica analítico-descriptiva. Cada equipo realizó su salida al campo durante una jornada acompañado por un docente, tarea que podía repetir, sin esa asistencia, en función de las necesidades y características de su TCU, práctica que emergió como novedosa para conocer el caso de estudio - "Raro pero necesario, es cuando uno realmente termina de comprender el espacio" ${ }^{22}$. Desde el inicio del curso se propició un intercambio más próximo, en clase, al interior de los grupos, entre grupos y con el equipo docente, respecto a las dificultades y desafíos percibidos al experimentar las áreas de estudio, aplicar los contenidos teóricos a la práctica de campo y superar las incertidumbres producto de abordar entornos y usuarios desconocidos y diversos a los de su cotidianeidad, hecho que motivó el agrado de los alumnos - "Cosas que me gustaron... enfocarnos en un mismo caso de estudio durante todo el cuatrimestre" ${ }^{13}$-.

Asimismo, se promovieron ámbitos de debate y participación en el aula-taller y la elaboración de producciones escritas y gráficas durante el proceso de análisis de los espacios verdes públicos-caso, dispositivos que contribuyeron a fortalecer la mirada crítica de los estudiantes, impactando positivamente en su capacidad reflexiva y habilidades para visibilizar e identificar las problemáticas del paisaje urbano y sobre los siguientes saberes:

\footnotetext{
${ }^{12}$ Encuesta 02 (2017).

${ }^{13}$ Encuesta 07 (2017).
} 
- Sitio y situación del estudio de caso;

- Interjuegos de escalas geográficas (espacio verde-caso, barrio, comuna, ciudad);

- Mutaciones estructurales y funcionales del paisaje;

- Observación y registro de aspectos físicos y prácticas sociales del espacio verde público (componentes vegetales, artefactuales, usos, rutinas, desplazamientos);

- Paisajes y cotidianeidad. Percepciones, valoraciones, sentido de lugar;

- Realización de encuestas y entrevistas; y

- Aplicación de la información producida en el TCU para elaborar la propuesta proyectual paisajística en el contexto del TIF.

\section{APORTES AL ABORDAJE PROYECTUAL DESDE LA DIDÁCTICA DEL PAISAJE}

El TCU demanda la participación activa de los estudiantes en el aprendizaje de saberes y su aplicación a situaciones de la realidad y permite reflexionar y prestar atención al contextualizar, descontextualizar y recontextualizar el paisaje desde la perspectiva geográfica, constituyendo una estrategia didáctica de “...planeación y puesta en práctica creada con el objeto de promover el mejoramiento institucional de (...) la enseñanza y/o de sus resultados" (Litwin, 2008, p. 65). En tal sentido, uno de los testimonios destaca la importancia de la asignatura y del proceso de enseñanza-aprendizaje abordando ámbitos reales y ajenos a la cotidianeidad estudiantil, señalando: -“[e]sta materia te hace bajar mucho a tierra y ves situaciones y contextos que uno no está acostumbrado. Siempre nos enseñaron desde lo ficticio y nos dejan volar. Esta materia te muestra la realidad"14-. La evaluación como parte del proceso didáctico representa para los estudiantes “...una toma de conciencia de los aprendizajes adquiridos..." - "Creo que fue fundamental el conocer con nuestros propios ojos el lugar" 15 , y para los docentes constituye "...una interpretación de las implicancias de la enseñanza en los aprendizajes" (Camilloni et al., 1998, p. 16), haciendo que “...aprendan, [y] ayudándoles a mejorar sus estrategias..." (Hargreaves et al., 2001, p. 84), es decir un recurso para perfeccionar los procesos de la enseñanza-aprendizaje.

\footnotetext{
${ }^{14}$ Encuesta 06 (2017).

${ }^{15}$ Encuesta 01 (2017).
} 

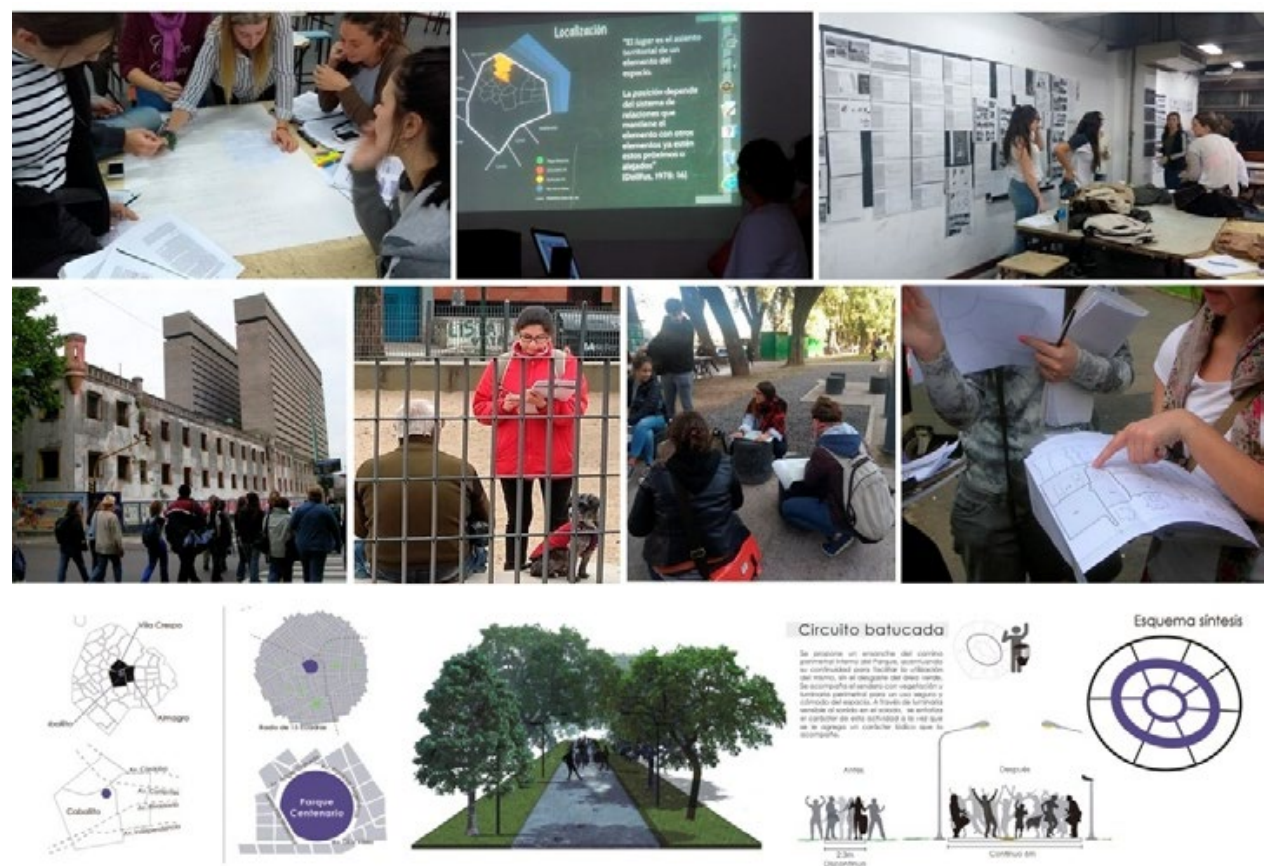

FIgURA 2. Proceso enseñanza aprendizaje. Fuente: Elaboración propia en base al archivo de la asignatura y TIF "Parque Centenario": Alumnas Budzisñki, N.; Romano, B. \& Rusca S. (2016)

Producto de la trayectoria del TCU, desde una mirada prospectiva, nos preguntamos cómo mejorar esta experiencia didáctica propicia para analizar críticamente el paisaje y articular las prácticas y percepciones comunitarias en el abordaje proyectual del espacio verde urbano, con la intención de potenciar esta "enseñanza poderosa" que "...crea una propuesta original que nos transforma como sujetos y cuyas huellas permanecen..." (Maggio 2012, p. 46), para lo cual efectuamos los siguientes aportes:

Estrategia didáctica: Integrar sincrónicamente durante el curso la estrategia didáctica de la práctica de campo con la elaboración del TCU del TIF, permitió abordar los desafíos y riesgos de recrearla en forma dinámica y con originalidad al detectar tempranamente falencias y/o dificultades de los estudiantes en el proceso de enseñanza-aprendizaje y desarrollo de sus actividades. En línea con la concepción de "integración" de Litwin (2008),${ }^{16}$ como punto de partida para favorecer la fusión de contenidos, la articulación del TCU con la práctica de campo del TIF, mediante el diseño de una didáctica de formas

${ }^{16}$ La autora entiende por "estrategia de integración” en la enseñanza “...las propuestas de actividades dirigidas a la conformación de un todo o una estructura y a la relación de sentido entre temas, conceptos o campos" (Litwin, 2008, p. 70). 
alternadas que aunó desafíos en el estudio de caso, permitió abordar su análisis de modo gradual, secuencial y profundo. Considerar los puntos de inflexión que marcaron cambios significativos en la trayectoria de la asignatura, nos condujo a repensar activamente el rol del TCU, apreciar su ductilidad y trascender aspectos repetitivos y rutinarios de la práctica docente, abordando su actualización como estrategia didáctica, en una sociedad donde “...el conocimiento es un recurso flexible, fluido, en constante expansión y movimiento (...) [y junto a] la creatividad y la inventiva son intrínsecos a lo que la gente hace" (Hargreaves, 2003, p. 27).

Práctica proyectual: Realizar las tareas del TCU durante el curso, implicó planificar, implementar, evaluar y ajustar una dinámica de enseñanza-aprendizaje y espacios de reflexión -entre alumnos, con los docentes y entre grupos- en relación a la indagación, análisis, diagnóstico y propuesta proyectual del estudio de caso del TIF. Estas actividades contribuyeron a reforzar el andamiaje reflexivo del estudio de caso y del paisaje. El TCU como experiencia didáctica “...organizada para investigar y resolver problemas que se presentan enredados en el mundo real" (Torp y Sage, 1999, p. 37) constituye una propuesta constante para los alumnos de comprometerse y abordar situaciones no estructuradas en la práctica proyectual del paisaje, estimulando ambientes de aprendizaje que profundizan la indagación y comprensión de contextos problemáticos. Siguiendo a García de la Vega (2012), el paisaje permite explorar su aprendizaje como escenario y secuencia geográfica, es decir, como representación de una única situación real o de diferentes momentos de su evolución. Programar y desarrollar las actividades de campo desde el inicio del curso, potenció preguntas, interacciones y seguimientos en torno a sus tareas y a la aplicación en su realización de los conceptos teóricos trabajados en clase, circunstancia que permitió generar sucesivos ajustes y restructuraciones comunicacionales, potenciando el feedback entre alumnos, grupos y equipo docente.

Construcción y comprensión del saber: En cada disciplina "[1]a difícil y compleja construcción del saber práctico es (...) dotar de sentido teórico o conceptualizar esos saberes construidos en una experiencia..." (Litwin, 2008, p. 32). Con la finalidad de provocar "actividades de comprensión"17, estimulamos el trabajo conjunto de los alumnos dentro del TCU y en el aula-taller, mediante el ejercicio de leer, entender y experimentar la bibliografía de cada unidad, aspecto en el cual, siguiendo a Perkins, procuramos alcanzaran la comprensión de contenidos “....no como un estado de posesión sino como un estado de capacitación. [dado que] Cuando entendemos algo, no sólo tenemos información sino que somos capaces de hacer ciertas cosas con ese conocimiento" (1995, p. 82). A la vez, elaborar propuestas paisajísticas abordando en campo un estudio de caso, como

${ }^{17}$ En términos de Perkins las “...cosas que podemos hacer, que revelan comprensión y la desarrollan" (1995, p. 82). 
sitio concreto del territorio, permitió a los estudiantes adquirir, comprender e interpretar la información en un contexto teórico-empírico, caracterizado por la aproximación subjetiva al objeto de estudio, a las prácticas y representaciones de sus usuarios y a los modos del habitar; experiencia personal que llevada al aula taller, favoreció el cotejo e intercambio de información con el conjunto, estimulando la construcción de conocimiento y enriqueciendo los insumos para planificar y diseñar el paisaje desde un ejercicio proyectual superador de contextos ficcionales.

Producción y proyecto colectivos: La eficacia del proceso de enseñanza-aprendizaje se potenció en la trayectoria de la asignatura y del TCU con la labor colaborativa y de intercambio que generó diversidad de motivaciones y dinámicas grupales. La escucha y el debate constituyeron elementos que activaron notablemente la mirada en perspectiva y contribuyeron a "enseña[r] a cambiar puntos de vista" (Maggio, 2012, p. 50) y promover instancias para crear, diseñar y formular propuestas. Las producciones grupales dieron cuenta de las circunstancias, vivencias y dificultades que rodearon la experiencia de campo, hecho que motivó instancias de reflexión crítica sobre los intercambios, impresiones, actividades y mecanismos para la resolución de problemas en el TCU, en cuyo marco se ejercitó la expresión escrita y el lenguaje técnico junto a las creaciones gráficas, a fin de que la impronta proyectual no resultara un óbice para su desarrollo. Las producciones mediadas por la tecnología: “...ofrecen otros usos, tales como presentar materiales nuevos que reorganizan la información, tender puentes para favorecer comprensiones, ayudar a reconocer la información en contextos diferentes; pero es fundamental reconocer que cuando las empleamos, ellas nos marcan límites concretos, formas de uso más adecuadas, requieren tiempos y condicionan las experiencias..." (Litwin, 2005, p. 20). Así, el TCU constituyó un insumo relevante para elaborar una propuesta paisajística viable para el estudio de caso del TIF, instancia en que cada equipo presentó y expuso su producción digitalizada a consideración del cuerpo docente y los otros grupos del curso.

Recursos tecnológicos e información confiable: Los cambios provocados por Internet imponen especial atención a su impacto en el proceso de enseñanza-aprendizaje y a desarrollar acciones para dotar de conocimientos a los estudiantes a fin de localizar sitios relevantes y confiables y fomentar el uso adecuado y análisis crítico de los recursos disponibles en la red. Aprender se refiere "...al aprendizaje de habilidades con las cuales aprender contenidos" (Monereo et al., 1994, p. 31) y, en tal sentido, esta herramienta puede resultar útil para acceder a información, datos estadísticos, planos, fotografías e imágenes satelitales que permitan una aproximación preliminar al espaciocaso, los componentes de su paisaje, escala geográfica y área de emplazamiento. Las tecnologías "...no sólo constituyen un conjunto de herramientas, sino un entorno (...) en el cual se producen las interrelaciones humanas" (Burbules y Callister, 2001, p. 19). Estas circunstancias nos llevaron a promover el empleo de medios alternativos en la labor 
de campo, activando la utilización de la telefonía celular que, por su accesibilidad y portabilidad, resultó fundamental para capturar y registrar las características del paisaje en estudio, permitiendo compartirlas y examinarlas desde diversas perspectivas. La información audiovisual amplificó la cantidad y diversidad de datos colectados, mediante los sonidos y movimientos presentes, lo que permitió incorporar aspectos dinámicos y reproducir la mirada de campo en el aula-taller. A su vez, implementar un espacio virtual de la asignatura, significó disponer de un entorno compartido entre docentes/estudiantes en sintonía con la dinámica del curso y de la enseñanza-aprendizaje, facilitando el trabajo colaborativo e interacción durante el TCU, además de permitir optimizar la comunicación, distribución de contenidos, seguimiento de los tutoriales y desarrollo de actividades que requerían instancias de socialización.

Enseñanza articulada y aprendizaje persistente: Wassermann sostiene que "[p]ara crear es necesario ser capaz de concebir nuevas ideas, nuevas estructuras, nuevos diseños, nuevos procedimientos..." (2006, p. 238), en esa línea impulsamos el TCU como una estrategia didáctica del proceso de enseñanza-aprendizaje del paisaje desde la geografía, articuladora de acciones orientadas a afrontar desafíos, ${ }^{18}$ promover la creatividad y generar la transferencia de conocimientos, entendiendo la innovación como una necesidad y un modo de evitar la repetición mecánica de prácticas consideradas "seguras y probadas". Implementar esta triangulación permitió generar una dinámica que condujo a los estudiantes a explorar contextos y situaciones desconocidos, enfrentar el reto de posicionarse en el "lugar del otro", comprender las necesidades y deseos ciudadanos en torno al espacio verde público, y expandir su imaginación a fin de generar propuestas proyectuales capaces de brindar respuestas viables a las problemáticas y demandas sociales que operan sobre estos paisajes de la cotidianeidad, situación que requiere la intención de tener “....realmente al otro o los otros en su ser presente y específico y (...) ref[erirse] a ellos con la intención de establecer una relación mutua y vivencial entre él y los otros" (Lipsman, 1997: 61). En resumen, la articulación didáctica en cada instancia del TCU posibilitó sustraer de la labor rutinaria a docentes y estudiantes, impidiendo la inacción y el anclaje en zonas de confort, permitiendo experimentar y socializar en contextos reales y construir un conocimiento empírico que impactó en las vivencias de cada alumno como practicado/recordado e influyó sustancialmente en el diagnóstico y diseño de su propuesta proyectual, generando que lo aprendido y aplicado resultara una enseñanza potente y perdurable; tal como señala Maggio, "la potencia de la enseñanza aparece cuando ayuda a reconstruir lo conocido, lo pensado, lo aprendido previamente; cuando interpela el sentido común y el mal sentido en el acercamiento a la sabiduría (2012, p. 63).

${ }^{18}$ Triangulamos y abordamos conjuntamente "aventura, creatividad y servicio", aspectos que Wassermann (2006) cita como elementos que generan desafíos en los alumnos. 


\section{CONSIDERACIONES FINALES}

El desafío de transformar la estrategia didáctica del Trabajo de Campo Urbano y asociarla con su práctica en el marco del desarrollo del Trabajo Integrador Final, motivó nuestra reflexión desde la geografía y el ámbito universitario en torno a su rol, explorando la construcción de alternativas dirigidas a innovar las modalidades del proceso de enseñanza-aprendizaje y del campo proyectual del paisaje. En este aspecto, adquirió relevancia fundamental documentar la trayectoria, experiencias y opiniones de los estudiantes en torno a la asignatura, como insumos para pensar y decidir ajustes y revisiones que contribuyeran a generar enfoques alternativos en su enseñanza y aplicación proyectual. La duda constituyó un aspecto esencial y dinamizador del proceso de enseñanza-aprendizaje, un elemento que generó incertidumbres y, a la vez, estimuló a docentes y estudiantes a asumir la tarea conjunta de intercambiar y experimentar en la búsqueda del conocimiento. Mirar en perspectiva fue el sostén para animarnos a provocar cambios y rupturas en la convicción de la necesidad de empoderar y profundizar una formación proyectual innovadora del paisaje urbano, asociada a las prácticas, representaciones y deseos de quienes lo habitan, responsable con el ambiente y el espacio público y comprometida con mejorar la calidad de vida.

Evitar anclar el equipo docente y alumnos a zonas de confort, si bien por momentos no resultó sencillo, constituyó una decisión que promovió acciones que enriquecieron el trayecto de la asignatura. Los estudiantes, a través del TCU, ejercitaron el abordaje de la realidad desde una perspectiva social y crítica, observando, interactuando y reflexionando respecto a las problemáticas geográfico-paisajísticas, extrapolando experiencias a otros contextos de análisis y en el marco de la generación de propuestas proyectuales viables. El desarrollo del TCU en un escenario marcado por la impronta de la instantaneidad y fluidez de la tecnología sobre los modos de informar y comunicar, emerge como una estrategia didáctica que revaloriza las prácticas in situ, la percepción y subjetividad del observador y la proximidad con el objeto de estudio, promoviendo un modo de proyectar el paisaje urbano con la comunidad usuaria, en el cual el diseño paisajístico no queda reducido únicamente a agregar valor estético o económico al espacio público, sino que además valoriza sus usos y resulta un nexo para fomentar los vínculos, identidad y pertenencia de quienes lo vivencian cotidianamente. Estas características distancian al TCU de ciertas herramientas que fijan la mirada proyectual exclusivamente en un diseño basado en entornos simulados, recurso que, si bien puede ser utilizado de manera complementaria, no debe sustituir o relegar la práctica de campo como experiencia directa para construir conocimiento y comprender la realidad espacial y social del territorio y el paisaje.

Estimular el "pensamiento apasionado" (Litwin, 2008) es incentivar la vocación de los estudiantes por articular la recopilación, análisis, creación y emisión de juicios 
valorativos a fin de desarrollar sus habilidades y la construcción y comprensión de saber, circunstancias que requieren ineludiblemente contar con docentes activos, reflexivos y que propongan actividades novedosas en las prácticas de la enseñanza superior y que, poniendo su mirada en el futuro, potencien la enseñanza proyectual del paisaje desde la perspectiva geográfica como un espacio de participación, que contribuya a ejercitar y promover los vínculos activos de diálogo entre sociedad y Universidad, como andamiaje sustancial de la formación disciplinar.

\section{BIBLIOGRAFÍA}

Besse, J. M. (2006). Las cinco puertas del paisaje. Ensayo de una cartografía de las problemáticas paisajeras contemporáneas. In J. Maderuelo (Dir.) Paisaje y pensamiento (pp. 45-171). Madrid: Abada Editores.

Burbules, N. \& Callister, T. (2001). Educación: riesgos y promesas de las nuevas tecnologías. Barcelona: Granica.

Dollfus, O. (1978) El análisis geográfico. Barcelona: Oikos-Tau.

Campari, G. (2014). La salud de nuestros jardines. Revista IF del Centro Metropolitano de Diseño (CMD), 9, 88-90.

Campari, G. (2015). Territorios urbanos de salud: observaciones en el espacio verde intrahospitalario. In Coordenadas contemporáneas de la sociología: tiempos, cuerpos, saberes (pp. 1-13). Actas XI Jornadas de Sociología de la UBA, Facultad de Ciencias Sociales, Universidad de Buenos Aires, 13 al 17 de julio 2015. Retrieved from http://www.aacademica.org/000-061/291

Campari, G.; Giacoia, G. \& Salerno, N. (2014). La resignificación del jardín hospitalario en la dinámica territorial de Buenos Aires. In C. A. Abate, et al., L. Karol (Comp.) UPE 11 Las Transformaciones urbanas: un debate sobre direcciones, orientaciones, estrategias y políticas que modelan la ciudad futura (pp. 645653). Actas del $11^{\circ}$ Simposio de la Asociación Internacional de Planificación y Ambiente, Universidad Nacional de La Plata, Buenos Aires, 16 al 19 de septiembre 2014. Retrieved from http://sedici.unlp.edu.ar/handle/10915/55044

Camilloni, A.; Celman, S.; Litwin, E. \& Palou de Mate, M. (1998). La evaluación de los aprendizajes en el debate didáctico contemporáneo. Buenos Aires: Paidós.

Capel, H. \& Urteaga, L. (1982). Las nuevas geografías. Barcelona: Editorial Salvat.

Consejo de Europa (2000). Convenio Europeo del Paisaje. Florencia. Retrieved from https://www.boe.es/boe/dias/2008/02/05/pdfs/A06259-06263.pdf

Estébanez, J. (1995). Los espacios urbanos. In R. Puyol; J. Estébanez \& R. Méndez Geografía humana (pp. 357-394). Madrid: Ediciones Cátedra. 
Feldman, D. (1999). Ayudar a enseñar. Buenos Aires: Aique.

García de la Vega,A. (2012). Un enfoque innovador en la didáctica del paisaje: escenario y secuencia geográfica. In R. de Miguel González; M. L. de Lázaro \& Torres; M. J. Marrón Gaite (Edits.) La educación geográfica digital (pp. 455-469). Zaragoza: Universidad de Zaragoza y Grupo de Didáctica de la Geografía de la Asociación de Geógrafos Españoles. Retrieved from http://didacticageografia.age-geografia. es//docs/Publicaciones/2012 Educacion Digital.pdf

Guber, R. (2004). El salvaje metropolitano. Buenos Aires: Paidós.

Hargreaves, A.; Earl, L.; Moore, S. \& Manning, S. (2001). Aprender a cambiar. La enseñanza más allá de las materias y los niveles. Barcelona: Octaedro.

Hargreaves, A. (2003). Enseñar para la sociedad del conocimiento. Educar para la creatividad. Barcelona: Ediciones Octaedro.

Libedinsky, M. (2001). La innovación en la enseñanza: Diseño y documentación de experiencias de aula. Buenos Aires: Paidós.

Liceras Ruiz. A. (2018). Los itinerarios didácticos en la enseñanza de la Geografía. Reflexiones y propuestas acerca de su eficacia en educación. UNES, 5, 66-81. Retrieved from https://www.revistaunes.com/index.php/revistaunes/article/ view/64/52

Lynch, K. (1998). La imagen de la ciudad. Barcelona: Editorial Gustavo Gilli.

Lipsman, M. (1997). Pensamiento complejo y educación. Ediciones de la Torre. Madrid.

Litwin, E. (Comp.) (2005). Tecnologías educativas en tiempos de Internet. Buenos Aires: Amorrortu.

Litwin, E. (2008). El oficio de enseñar. Buenos Aires: Paidós.

Maggio, M. (2012). Enriquecer la enseñanza: los ambientes con alta disposición tecnológica como oportunidad. Buenos Aires: Paidós.

Monereo, C. (Coord.); Catelló, M.; Clariana, M.; Palma, M. \& Luïsa Pérez, M. (1994). Estrategias de aprendizaje y enseñanza. Formación del profesorado y aplicación en la escuela. Barcelona: Graó.

Nogué, J. (Ed.) (2009). La construcción social del paisaje. Madrid: Biblioteca Nueva.

Ortega Valcárcel, J. (2007). La geografía para el siglo XXI. In J. Romero (Coord.) Geografía humana (pp. 27-55). Barcelona: Editorial Ariel.

Perkins, D. (1995). La escuela inteligente. Barcelona: Gedisa.

Piñeiro Peleteiro, M. del R. (1997). El pensamiento geográfico y el trabajo de campo en el siglo XX. Didáctica Geográfica, 2, 25-31.

Romero, R. R. (1987). Grupo. Objeto y Teoría. Buenos Aires: Lugar Editorial. 
Sanchez Ogallar, A. (1997). La observación e interpretación del paisaje. Didáctica Geográfica, 2, 45-55.

Santos, M. (1996). Metamorfosis del espacio habitado. Barcelona: Oikos-Tau.

Souto, M. (2006). El carácter de "artificio" del dispositivo pedagógico en la formación para el trabajo. Buenos Aires: Universidad de Buenos Aires.

Torp, L. \& Sage, S. (1999). El aprendizaje basado en problemas. Buenos Aires: Amorrortu.

Vasilachis de Gialdino, I. (Coord.) (2006). Estrategias de investigación cualitativa. Barcelona: Editorial Gedisa.

Varela, B. \& Campari, G. (2009). La importancia del Trabajo de Campo para las ciencias sociales en la pedagogía universitaria. In E. Litwin, et al. Libro de Resúmenes Primer Congreso Internacional de Pedagogía Universitaria (pp. 810-811), Facultad de Derecho, Universidad de Buenos Aires, 7 al 9 de septiembre 2009. Buenos Aires: Eudeba.

Varela, B. \& Campari, G. (2012). De la teoría al Trabajo de Campo con estudiantes universitarios. In L. Soria y B. Goldwaser, (Eds. \& Comps.), Problemáticas Geográficas Argentinas. Territorios - Actores Sociales - Sistemas productivos (pp. 317-343). San Miguel: Gepse Ediciones.

Wasserman S. (1999). El estudio de casos como método en la enseñanza. Buenos Aires: Amorrortu.

Zusman, P. (2011). La tradición del trabajo de campo en Geografía. Geograficando, 7 (7), 15-32. Retrieved from http://www.memoria.fahce.unlp.edu.ar/art revistas/ pr.5089/pr.5089.pdf 